\title{
SELF-DUAL LATTICES FOR MAXIMAL ORDERS IN GROUP ALGEBRAS
}

\author{
DAVID GLUCK
}

\begin{abstract}
Let $G$ be a finite group and $V$ an irreducible $\mathbf{Q}[G]$-module. Let $R$ be a Dedekind domain with quotient field $\mathbf{Q}$ such that $|G|$ is a unit in $R$. For applications to topology it is of interest to know if $V$ contains a full self-dual $R[G]$-lattice. We show that such lattices always exist for some major classes of finite groups.
\end{abstract}

Let $G$ be a finite group and let $R$ be a Dedekind domain with quotient field $\mathbf{Q}$ such that $|G|$ is a unit in $R$. We say that a $\mathbf{Q}[G]$-module $V$ is balanced if $V$ contains a full self-dual $R[G]$-lattice. For applications to surgery theory (see [10, p. 28-36]) it is of interest to establish criteria for balance.

We show that any irreducible $\mathbf{Q}[G]$-module is balanced when $G$ is $p$-hyperelementary for an odd prime $p$, when $|G|$ is odd, or when $G$ is a 2-group. We know of no example of an unbalanced $\mathbf{Q}[G]$-module for any finite group $G$. Theorem 3, our main criterion for balance, follows easily from standard but deep results in integral representation theory.

I would like to thank Bruce Williams for bringing this problem to my attention.

Preliminaries. For $G$ and $R$ as above, $R[G]$ is a maximal order in $\mathbf{Q}[G]$ by [9, Theorem 41.1]. Therefore every left $R[G]$-lattice $L$ is projective, and $L$ is indecomposable if and only if $\mathbf{Q} L$ is an irreducible $\mathbf{Q}[G]$-module [9, Corollary 21.5]. The central primitive idempotents $e_{i}(1 \leq i \leq m)$ of $\mathbf{Q}[G]$ lie in $R[G]$ by $[\mathbf{9}$, Theorem 10.5], and any $R[G]$-lattice $L$ decomposes as $L=e_{1} L \oplus \cdots \oplus e_{m} L$. If $L$ and $M$ are isomorphic $R[G]$-lattices, so are $e_{i} L$ and $e_{i} M$ for each $i$. By $[\mathbf{9}$, Theorems 11.1 and 18.7] two $R[G]$-lattices $L$ and $M$ belong to the same genus if and only if $\mathbf{Q} L \cong \mathbf{Q} M$. If $H \leq G$ and $L$ is an $R[H]$-lattice, then $L^{G}$ denotes the induced lattice $R[G] \otimes_{R[H]} L$.

If $L$ is a left $R[G]$-lattice, then $L^{*}$ denotes the dual (contragredient) left $R[G]$ lattice. If $L$ affords the matrix representation $\rho: G \rightarrow \operatorname{GL}(n, R)$, then $L^{*}$ affords the composition of $\rho$ with the inverse transpose automorphism of $\operatorname{GL}(n, R)$. In particular, $L \cong L^{* *}$. For $e_{i}$ as above, $e_{i} L^{*} \cong\left(e_{i} L\right)^{*}$. If $L$ is an $R[H]$-lattice for some $H \leq G$, then $\left(L^{*}\right)^{G} \cong\left(L^{G}\right)^{*}$.

If $V$ is a $\mathbf{Q}[G]$-module, let $\chi_{V}$ denote the character of $V$. If $\chi$ is an irreducible complex character of $G$, let $m(\chi)$ denote the Schur index of $\chi$ over $\mathbf{Q}$, and let $\operatorname{Tr}(\chi)$ denote the sum of the distinct algebraic conjugates of $\chi$. If $\psi$ is any rational-valued character of $G$, let $p(\psi)$ be the permutation index of $\psi$-the least integer $p$ such that $p \psi$ is an integral linear combination of permutation characters of $G$. By $[\mathbf{4}$, Theorem 5.21] $p(\psi)$ divides $|G|$.

Received by the editors April 5, 1984.

1980 Mathematics Subject Classification. Primary 20C10; Secondary 20C15. 
The following lemmas contain the key results we need from integral representation theory. If $M$ and $N$ are $R[G]$-lattices, we write $M \mid N$ to mean that $M$ is isomorphic to a direct summand of $N$.

LEMMA 1. Let $V$ be an irreducible $\mathbf{Q}[G]$-module. Let $M$ and $N$ be $R[G]$-lattices such that $\mathbf{Q} M \cong m V$ and $\mathbf{Q} N \cong n V$, with $m<n$. Then $M \mid N$.

Proof. Let $M=M_{1} \oplus \cdots \oplus M_{m}$ and $N=N_{1} \oplus \cdots \oplus N_{n}$ be decompositions of $M$ and $N$ as direct sums of indecomposable lattices. Since $M_{1}, N_{1}$, and $N_{2}$ belong to the same genus, we may write $N_{1} \oplus N_{2} \cong M_{1} \oplus L_{1}$ for an indecomposable $R[G]$-lattice $L_{1}$ by $\left[\mathbf{9}\right.$, Corollary 27.3]. Let $M^{\prime}=M_{2} \oplus \cdots \oplus M_{m}$ and let $N^{\prime}=$ $L_{1} \oplus N_{3} \oplus \cdots \oplus N_{n}$. Then $M \cong M_{1} \oplus M^{\prime}$ and $N \cong M_{1} \oplus N^{\prime}$. By induction on $m$ we may assume $M^{\prime} \mid N^{\prime}$. Hence $M \mid N$.

We say that a $\mathbf{Q}[G]$-module $V$ is Eichler if no simple component of $\operatorname{End}_{\mathbf{Q}[G]} V$ is a totally definite quaternion algebra, as defined in $[\mathbf{9}$, p. 293]. We note that if $V$ is an irreducible $\mathbf{Q}[G]$-module which is not Eichler, and $\chi$ is an irreducible complex constituent of $\chi_{V}$, then $\chi(1)=m(\chi)=2$. The structure of $G / \operatorname{Ker} V$ is severely restricted; see $[\mathbf{9}$, p. 344]. We say that an $R[G]$-lattice $L$ is Eichler if $\mathbf{Q} L$ is Eichler.

LEMMA 2. Let $X, M$, and $N$ be $R[G]$-lattices. If $X \oplus M \cong X \oplus N$ and $M$ is Eichler, then $M \cong N$.

PROOF. See [5, p. 14].

\section{The main criterion.}

THEOREM 3. Let $V$ be an irreducible $\mathbf{Q}[G]$-module. Suppose $p\left(\chi_{V}\right)$ is odd and $V$ is Eichler. Then $V$ is balanced.

Proof. Let $p=p\left(\chi_{V}\right)$. Then $p V \oplus V_{1} \cong V_{2}$ for $\mathbf{Q}[G]$-permutation modules $V_{1}$ and $V_{2}$. Let $L_{1}$ and $L_{2}$ be full self-dual $R[G]$-lattices in $V_{1}$ and $V_{2}$, respectively. Let $e$ be the primitive central idempotent in $\mathbf{Q}[G]$ which corresponds to $V$. Then $e L_{1}$ and $e L_{2}$ are full self-dual $R[G]$-lattices in $e V_{1}$ and $e V_{2}$, respectively. By Lemma 1 we may write $e L_{2}=e L_{1} \oplus L_{0}$ for an $R[G]$-lattice $L_{0}$ with $\mathbf{Q} L_{0} \cong p V$. Taking duals yields $e L_{2} \cong e L_{1} \oplus L_{0}^{*}$. By Lemma 2 we have $L_{0} \cong L_{0}^{*}$.

Now let $M$ be a fixed $R[G]$-lattice with $\mathbf{Q} M=V$. Let $M_{0}=\frac{1}{2}(p-1)\left(M \oplus M^{*}\right)$. By Lemma 1 we may write $L_{0} \cong M_{0} \oplus M_{1}$, where $\mathbf{Q} M_{1} \cong V$. Since $M_{0}^{*} \cong M_{0}$ and $L_{0}^{*} \cong L_{0}$. we have $L_{0} \cong M_{0} \oplus M_{1}^{*}$. Lemma 2 yields $M_{1} \cong M_{1}^{*}$.

We recall that a group $G$ is called $p$-hyperelementary if $G$ has a cyclic normal $p$-complement.

COROLlary 4. Suppose $G$ is p-hyperelementary for an odd prime $p,|G|$ is odd, or $G$ is abelian. Then every irreducible $\mathbf{Q}[G]$-module $V$ is balanced.

PROOF. Suppose first that $G$ is $p$-hyperelementary for an odd prime $p$. Let $\chi$ be an irreducible complex constituent of $\chi_{V}$. By [4, Theorem 6.15] $\chi(1)$ is odd, so $V$ is Eichler. By [6, Definition 1.6 and Proposition 7.2] $\chi_{V}$ has odd permutation index. Hence, $V$ is balanced by Theorem 3. A similar argument works if $|G|$ is odd.

If $G$ is cyclic, then $G$ is $p$-hyperelementary for any odd prime $p$, so $V$ is balanced. If $G$ is abelian, then $G / \operatorname{Ker} V$ is cyclic, so $V$ is balanced. 
COROLlaRY 5. Let $G$ be a finite group and $V$ an irreducible $\mathbf{Q}[G]$-module. If $V$ is Eichler and $V_{H}$ is balanced for every 2-hyperelementary subgroup $H$ of $G$, then $V$ is balanced.

ProOF. Let $\nVdash$ be the family of all hyperelementary subgroups of $G$. By $[4$, Theorem 8.10 ] may write

$$
1_{G}=\sum_{H \in \mathcal{H}} a_{H} 1_{H}^{G}-\sum_{H \in \mathcal{H}} b_{H} 1_{H}^{G},
$$

where all the $a_{H}$ and $b_{H}$ are nonnegative integers. Then

$$
\chi_{V}=\sum a_{H}\left(\left.\chi_{V}\right|_{H}\right)^{G}-\sum b_{H}\left(\left.\chi_{V}\right|_{H}\right)^{G} .
$$

Hence,

$$
V \oplus \bigoplus_{H \in \mathcal{H}} b_{H}\left(V_{H}\right)^{G} \cong \bigoplus_{H \in \mathcal{H}} a_{H}\left(V_{H}\right)^{G} .
$$

By the hypotheses and Corollary $4, V_{H}$ and, hence, $\left(V_{H}\right)^{G}$ are balanced for all $H \in$ H. Let $L_{1}$ and $L_{2}$ be full self-dual $R[G]$-lattices in $\bigoplus b_{H}\left(V_{H}\right)^{G}$ and $\bigoplus a_{H}\left(V_{H}\right)^{G}$, respectively. The argument in the first paragraph of the proof of Theorem 3 shows that $V$ is balanced.

REMARKS. When $G$ is 2-hyperelementary and $V$ is an irreducible $\mathbf{Q}[G]$-module, there is a subgroup $H$ of $G$ and a primitive $\mathbf{Q}[H]$-module $W$ such that $V=W^{G}$. Let $\bar{H}=H / \operatorname{Ker} W$. Since every normal abelian subgroup of $\bar{H}$ is cyclic, an application of $\left[\mathbf{1 1}\right.$, Lemma 2.3] to $\mathbf{O}_{2}(\bar{H})$ shows that $\bar{H}$ contains a self-centralizing normal cyclic subgroup. Thus the question of whether $V$ is balanced reduces in a sense to a Galois action situation, as in the Brauer-Witt theorem on Schur indices; see [4, Theorem 10.7].

We also remark that Corollaries 4 and 5 do not exhaust the applications of Theorem 3. See $[\mathbf{2}, \mathbf{7}$ and $\mathbf{8}]$ for more information about permutation indices.

2-groups. We prove a strong form of the balance property for 2-groups.

Proposition 6. Let $G$ be a 2-group and let $V$ be an irreducible $\mathbf{Q}[G]$-module. Then $V$ contains a full self-dual $\mathbf{Z}[G]$-lattice.

Proof. Let $G$ be a 2-group with a faithful irreducible primitive $\mathbf{Q}[G]$-module $V$. To prove the proposition, it suffices to show that $|G| \leq 2$. Since $G$ has no noncyclic normal abelian subgroup, [3, Theorem 5.4.10] shows that $G$ is cyclic, dihedral, semidihedral, or generalized quaternion. Also, $G \neq D_{8}$ and we may assume $G \neq 1$.

Suppose $G$ is not cyclic and $|G|\rangle 8$. Let $\langle x\rangle$ be the maximal cyclic subgroup of $G$ and choose $t \in G$ so that $G=<t, x>$. If $G$ is not generalized quaternion, choose $t$ to be an involution. Let $G_{0}=<t, x^{2}>$. Let $\lambda$ be a faithful linear character of $\langle x\rangle$ and let $\lambda(x)=\epsilon$. Then $\epsilon$ is a primitive $2^{n}$ th root of 1 for some $n \geq 3$. Let $\chi=\lambda^{G}$ and let $\chi_{0}=\left.\chi\right|_{G_{0}}=\left(\left.\lambda\right|_{<x^{2}>}\right)^{G_{0}}$. Then $\chi$ and $\chi_{0}$ are irreducible complex characters of $G$ and $G_{0}$, respectively.

The field of values $\mathbf{Q}\left(\chi_{0}\right)$ is contained in $\mathbf{Q}\left(\epsilon^{2}\right)$, while $\chi(x)=\epsilon+\epsilon^{-1}$ or $\epsilon+$ $\epsilon^{2^{n-1}-1}$. Let $\sigma$ be the unique nonidentity field automorphism of $\mathbf{Q}(\epsilon)$ which fixes $\epsilon^{2}$. Then $\epsilon^{\sigma}=\epsilon^{2^{n-1}+1}=-\epsilon$. Hence, $\chi(x)^{\sigma}=-\chi(x) \neq 0$, so that $\chi^{\sigma} \neq \chi$, $\chi_{0}^{G}=\chi+\chi^{\sigma}$, and $\left[\mathbf{Q}(\chi): \mathbf{Q}\left(\chi_{0}\right)\right]=2$. By $\left[\mathbf{1}, 11.7\right.$ and 11.8] we have $m(\chi)=m\left(\chi_{0}\right)$. 
Therefore $\left(m\left(\chi_{0}\right) \operatorname{Tr} \chi_{0}\right)^{G}=m(\chi) \operatorname{Tr}(\chi)$. It follows that $V$ is induced from an irreducible $\mathbf{Q}\left[G_{0}\right]$-module, contrary to assumption.

Thus $G=Q_{8}$ or $G$ is cyclic. In these cases $G$ has a unique faithful irreducible $\mathbf{Q}[G]$-module which is induced from the unique subgroup of $G$ of order 2. Since $V$ is primitive, $|G|=2$.

FINAL REMARK. The referee has pointed out that it would be more significant to discuss whether a $\mathbf{Q}[G]$-module contains a self-dual $\mathbf{Z}[G]$-lattice rather than a self-dual $R[G]$-lattice. For the application to topology, however, our results on $R[G]$-lattices are significant. They show that, for appropriate $G$, the computation of relative surgery obstruction groups arising in a certain long exact sequence reduces to the computation of surgery obstruction groups for maximal orders in division algebras.

\section{REFERENCES}

1. W. Feit, Characters of finite groups, Benjamin, New York, 1967.

2. D. Gluck, A characterization of generalized permutation characters, J. Algebra 63 (1980), 541-547.

3. D. Gorenstein, Finite groups, Harper and Row, New York, 1968.

4. I. M. Isaacs, Character theory of finite groups, Academic Press, New York, 1976.

5. H. Jacobinski, Genera and decomposition of lattices over orders, Acta Math. 121 (1968), 1-29.

6. T. Y. Lam, Artin exponent of finite groups, J. Algebra 9 (1968), 94-119.

7. J. Rasmussen, Rationally represented and permutation characters of nilpotent groups, J. Algebra 29 (1974), 504-509.

8. __ Artin index of faithful metacyclic groups, J. Algebra 46 (1977), 511-522.

9. I. Reiner, Maximal orders, Academic Press, London, 1975.

10. I. Hambleton, L. Taylor and B. Williams, An introduction to maps between surgery obstruction groups, preprint.

11. T. R. Wolf, Solvable and nilpotent subgroups of GL $\left(n, q^{m}\right)$, Canad. J. Math. 34 (1982), 10971111.

Department of Mathematics, Wayne State University, Detroit, Michigan 48202 